\section{Zivilarzt - Militärdienst}

Welches sind die häufigsten an die Sektion Militärärztlicher Dienst
der UG San gerichteten Fragen von seiten der praktizierenden Ärzte?

Generalstab, Untergruppe Sanität, Sektion Militärärztlicher Dienst

\section{Einleitung}

Die Sektion Militärärztlicher Dienst (MAD) der Untergruppe Sanität (UG San) ist die zentrale Anlaufstelle in militärmedizinischen Belangen, einerseits für die Schul- und Truppenärzte, andererseits aber auch für die Angehörigen der Armee und ihre behandelnden Zivilärzte (Anmerkung: Der Begriff "Zivilärzte» steht im folgenden für Ärzte wie auch für Ärztinnen!).

Die Sektion MAD besteht aus drei Kreisbüros mit je einem Kreisarzt, dem Rekrutierungsbüro und dem Chef der Sektion.

Im folgenden werden die häufigsten uns gestellten Fragen aufgeführt und kurz beantwortet. Die Fragen sind gegliedert nach administrativen und militärmedizinischen Aspekten.

\section{Administrative Fragen}

Verschiebung einer Dienstleistung: Ein Rekrut oder ein Angehöriger der Armee muss aus medizinischen Gründen seine Dienstleistung verschieben. An wen ist das Arztzeugnis zu senden?

Es muss zwischen Soldaten, Unteroffizieren und Offizieren unterschieden werden:

Bei den Soldaten muss ein Dienstverschiebungsgesuch aus medizinischen Gründen über die aufbietende Stelle eingeschickt werden. Bei kantonalen Truppen ist das entsprechende Kreiskommando, bei eidgenössischen Truppen die Untergruppe Personelles der Armee des Generalstabs zuständig.

Bei den Unteroffizieren und Offizieren ist das Dienstverschiebungsgesuch dem entsprechenden Einheitskommandanten zuzustellen.

In beiden Fällen sind die Arztzeugnisse in verschlossenen Umschlägen dem Dienstverschiebungsgesuch beizulegen. Die Arztzeugnisse müssen in jedem Falle durch die vorgenannten Stellen zur Beurteilung der Sektion MAD vorgelegt werden. Diese stellt ihrerseits der aufbietenden Stelle gegebenenfalls den Antrag auf Dispensation. Liegt das Arztzeugnis nicht verschlossen bei, kann die aufbietende Stelle in klaren Fällen (z.B. Unterschenkelfraktur, Hospitalisation usw.) ohne Rücksprache mit der Sek-

Korrespondenz: Dr. med. Rolf Huber

GST, UG San

Sektion MAD

CH-3003 Bern tion MAD eine Dispensation von der Dienstleistung aussprechen.

Bei kurzfristigen Gesuchen (14 Tage vor der Dienstleistung) ist es von Vorteil, zusätzlich eine Kopie des Dienstverschiebungsgesuches und des Arztzeugnisses direkt an die Sektion MAD zu senden, um so den administrativen Weg abzukürzen.

Medizinische Entlassung aus der Armee (Dienstuntauglichkeit): An wen sind Arztzeugnisse für die Entlassung eines Angehörigen der Armee aus der Militärdienstpflicht zu richten? Wie soll ein solches Zeugnis abgefasst sein?

Arztzeugnisse, die nicht die Dienstfähigkeit für einen bevorstehenden Dienst, sondern generell die Diensttauglichkeit betreffen, sind in jedem Fall direkt der Sektion MAD zuzustellen.

Die Sektion MAD wird dann den Angehörigen der Armee vor eine Untersuchungskommission (UC) aufbieten oder in klaren, gut dokumentierten Fällen den Entscheid "untauglich" durch die UC UG San in absentia fällen.

Die Arztzeugnisse werden immer von den Kreisärzten der Sektion MAD begutachtet und sollen entsprechend abgefasst werden. Alle involvierten Sachbearbeiter in der Sektion unterstehen dem Amtsgeheimnis.

Sowohl die Sektion MAD wie auch die Untersuchungskommissionen sind darauf angewiesen, dass sie vom behandelnden Arzt ein ausführliches Zeugnis erhalten. Entgegen ihrem Namen führen die meisten Untersuchungskommissionen keine Untersuchungen durch, sondern beurteilen den Patienten lediglich aufgrund der vorliegenden Akten und des persönlichen Gesprächs.

Im Minimum soll das Arztzeugnis deshalb eine kurze Anamnese, die genaue Diagnose, den Verlauf, die Therapie und eine Prognose beinhalten. Falls vorhanden, sollen zusätzlich die Resultate spezieller Untersuchungen (z.B. Befunde bildgebender Verfahren, Laborresultate, bei psychiatrischen Zeugnissen die Resultate der psychologischen Tests) enthalten sein. Geschätzt wird ebenfalls ein Antrag bezüglich Diensttauglichkeit aus der Sicht des behandelnden Arztes. Für die Abschätzung der Diensttauglichkeit helfen auch Angaben über die sportliche Leistungsfähigkeit des Angehörigen der Armee. Es sei erwähnt, dass die Untersuchungskommissionen das Arztzeugnis zu würdigen haben, jedoch in ihrem Entscheid über die Diensttauglichkeit frei sind.

Da die Archivierung der Sanitätsdossiers nach AHV-Nummer erfolgt, erleichtert uns die Angabe der AHV-Nummer auf den Zeugnissen die Arbeit wesentlich.

Beurteilungskriterien: Welches sind die Kriterien für eine Militärdiensttauglichkeit bzw. -untauglichkeit? Die Kriterien der Diensttauglichkeit sind im vertraulichen militärischen Dokument «Nosologia Militaris» enthalten. Diese Dokumentation kann wegen der vertraulichen Klassifizierung nicht an die Zivilärzte $a b-$ gegeben werden. 
Jedoch wurden in der Schweizerischen Ärztezeitung [1] Auszüge und die allgemeinen Richtlinien aus dieser Dokumentation veröffentlicht. Dieser Artikel kann bei der Sektion MAD als Sonderdruck bezogen werden.

Kostenträger: Für den Angehörigen der Armee soll ein ärztliches Zeugnis oder ein Gutachten ausgestellt werden. Wer kommt für die Kosten auf? Grundsätzlich gilt, dass ein Angehöriger der Armee, welcher ein medizinisches Zeugnis wünscht, auch Kostenträger ist.

Wenn die UG San eine Abklärung veranlasst, erhalten die praktizierenden Kollegen/-innen einen schriftlichen Auftrag. In diesem Fall ist die UG San Kostenträger. Der Zivilarzt muss dann nach UV/ MV/IV-Tarif abrechnen.

Zivilarzt als Truppenarzt: Ein Zivilarzt wird für die Betreuung der Truppe angefragt. Wie kann er sich über seine bevorstehende Aufgabe informieren? Beim Fehlen eines Truppenarztes für die medizinische Betreuung der Truppe im Friedensfall werden praktizierende Zivilärzte für die Übernahme dieser Funktion angefragt.

Die Truppe meldet Zivilärzte, welche diese Funktion übernehmen, der Sektion MAD, damit ihnen leihweise eine ausführliche Dokumentationsmappe zugestellt werden kann. Wenn die Meldung und somit die Zustellung nicht wie vorgesehen funktioniert, können die involvierten Zivilärzte direkt die entsprechenden Unterlagen anfordern.

Die erwähnte Dokumentationsmappe enthält Unterlagen zur Funktion des Zivilarztes im Truppenarztdienst (z.B. die Weisung Nr. 45 des Oberfeldarztes über die ärztliche Versorgung der Truppe durch Zivilärzte), in denen nebst den Aufgaben des Zivilarztes auch die finanzielle Entschädigung geregelt ist. Darüber hinaus liegen der Dokumentationsmappe die Beurteilungskriterien für die Diensttauglichkeit ("Nosologia Militaris») und eine Auswahl der wichtigsten Formulare bei.

Behandlungskosten: Behandlung von Angehörigen der Armee durch Zivilärzte während der obligatorischen Dienstleistungen. Wer bezahlt?

Die Angehörigen der Armee müssen sich im Falle von medizinischen Problemen während der Dienstleistungen grundsätzlich an den Truppenarzt wenden. Die militärischen Dienstleistungen umfassen auch den allgemeinen und persönlichen Urlaub. Nur bei einem Notfall im Urlaub soll sich der Angehörige der Armee an einen Zivilarzt wenden.

Die Militärversicherung deckt die ambulanten und stationären medizinischen Kosten, die während einer Dienstleistung entstehen. Stationäre Aufenthalte werden für alle Angehörigen der Armee gemäss Tarif für die Allgemeine Abteilung vergütet. Halbprivate oder private stationäre Aufenthalte werden subsidiär durch die zivilen Krankenkassen übernommen, sofern eine Zusatzversicherung vorhanden ist. Eine allfällig während der Dienstleistung sistierte private Krankenversicherung muss in diesem Fall wieder aktiviert werden.

$\mathrm{Ab}$ und zu ergibt sich am Wochenende die Situation, dass sich Angehörige der Armee auch bei Bagatellproblemen oder für eine "second opinion" an den Zivilarzt wenden. Rückfragen bei der Militärversicherung ergaben, dass auch diese Kosten durch die Militärversicherung übernommen werden.

Die Arztzeugnisse sind bei solchen Konsultationen dem Patienten zu Händen des Truppenarztes mitzugeben.

Waffenentzug: Einem psychisch angeschlagenen, selbst- oder fremdgefährdenden Patienten soll seine persönliche Waffe entzogen werden. Wie kann der behandelnde Arzt vorgehen?

Der behandelnde Arzt muss in diesem Fall über die örtliche Polizei die Konfiszierung der Waffe veranlassen.

Die Waffe wird von der Polizei gegen Beleg im zuständigen Zeughaus deponiert.

Gleichzeitig muss der Sektion MAD ein Antrag auf vorübergehende Dispensation vom Militärdienst bzw. auf Dienstuntauglichkeit gestellt werden.

Die Sektion MAD entscheidet dann aufgrund der medizinischen Akten, ob die Waffe dem Angehörigen der Armee definitiv abgenommen wird oder ob sie ihm zurückgegeben werden kann.

Zivildienst: Wie muss ein Arztzeugnis abgefasst werden, damit ein Angehöriger der Armee im Hinblick auf den zivilen Ersatzdienst beurteilt werden kann?

Für den zivilen Ersatzdienst (Zivildienst) muss der Angehörige der Armee zwingend diensttauglich für die Armee sein. Lediglich unter dieser Voraussetzung kann er ein Gesuch für das Leisten von Zivildienst stellen.

Im Gegensatz zu anderen Armeen (z.B. Deutschland) gibt es in der Schweiz keine freie Wahl zwischen Militärdienst und zivilem Ersatzdienst. Zivildienst leisten kann in der Schweiz nur derjenige, der glaubhaft darlegen kann, dass er den Militärdienst nicht mit seinem Gewissen vereinbaren kann. Die Gewissensgründe werden durch eine nicht medizinische Zulassungskommission beurteilt!

Zusammengefasst: Medizinische Kriterien gelten für die Beurteilung der Diensttauglichkeit. Die Diensttauglichkeit ist Voraussetzung für das Eintreten auf ein Zivildienstgesuch. Für die Zulassung zum Zivildienst gelten ethische Kriterien, jedoch keine medizinischen Fakten.

Anmerkung: Verwirrung stiften häufig die beiden Begriffe "Zivildienst" und "Zivilschutz». Der Zivildienst ist wie beschrieben der zivile Ersatzdienst. Der Dienst beim Zivilschutz ist die obligatorische Dienstleistung nach der Absolvierung des Militärdienstes, beziehungsweise beim Vorliegen von entsprechenden medizinischen Gründen anstelle des Militärdienstes. 
Tabelle 1

Dienstunfähigkeit nach Implantation von 0steosynthesematerial.

\begin{tabular}{ll}
\hline Lokalisation & Karenzzeit (Monate) \\
\hline Oberarm & $6-12$ \\
\hline Vorderarm & $4-6$ \\
\hline Wirbelsäule & 12 \\
\hline Oberschenkel & $12-18$ \\
\hline Unterschenkel und Fuss & $6-12$ \\
\hline
\end{tabular}

Tabelle 2

Dienstunfähigkeit nach Entfernung des Osteosynthesematerials.

\begin{tabular}{ll}
\hline Lokalisation & Karenzzeit (Monate) \\
\hline Obere Extremität & 3 \\
\hline Oberschenkel & 6 \\
\hline Unterschenkel und Fuss & 3 \\
\hline
\end{tabular}

\section{Medizinische Fragen}

Orthopädie: Gibt es Richtlinien über die Dienstfähigkeit nach orthopädischen Eingriffen?

Grundsätzlich gilt, dass sich die Beurteilung der Dienstfähigkeit nach operativen Eingriffen auf die Empfehlungen des Operateurs bzw. des nachbehandelnden Arztes stützt.

Liegendes Osteosynthesematerial stellt per se keinen Hinderungsgrund für die Dienstfähigkeit dar.

Liegen keine konkreten Empfehlungen des behandelnden Arztes vor, wird sich der Truppenarzt an folgende Richtlinien bezüglich Karenzzeiten nach Implantation (Tab. 1) bzw. Karenzzeiten nach Entfernen von Osteosynthesematerial (Tab. 2) halten.

Nach Ablauf dieser Karenzfristen ist der Angehörige der Armee wieder uneingeschränkt dienstfähig. Voraussetzung dafür sind selbstverständlich ein funktionell einwandfreies Resultat und das Fehlen von Weichteilproblemen (z.B. behindernde Narben).

Bei Unsicherheiten bezüglich der Dienstfähigkeit kann mit einem Kreisarzt der Sektion MAD Kontakt aufgenommen und das konkrete Vorgehen besprochen werden.

Epileptiker: Sind medikamentös gut eingestellte Epileptiker in gewissen Funktionen diensttauglich? Epileptiker sind grundsätzlich nicht diensttauglich. Ausnahmen sind lediglich Patienten, deren epileptische Anfälle sich auf das Kindesalter beschränkt haben.

Auch wenn der gut eingestellte Epileptiker den zivilen Fahrausweis erwerben kann, ist die Diensttauglichkeit nicht gegeben.
Positiver HIV-Befund: Sind HIV-positive Patienten grundsätzlich diensttauglich?

Stellungspflichtige und Rekruten mit positiver HIVSerologie unter Therapie sind grundsätzlich dienstuntauglich. Bei Angehörigen der Armee mit abgeschlossener Grundausbildung muss die Situation etwas differenzierter beurteilt werden. Die Beurteilung hängt vom medizinischen Verlauf, von der Therapie, der militärischen Funktion und der Einstellung des Angehörigen der Armee ab. Wir verweisen wiederum auf den Artikel in der Schweizerischen Ärztezeitung [1].

Methadonpatienten: Sind Angehörige der Armee unter Methadon bei gutem medizinischem und psychosozialem Verlauf im Ausnahmefall diensttauglich?

Patienten, welche harte Drogen konsumieren, in einem Drogenentzug stehen (inklusive Substitutionsprogramm), oder einen Drogenentzug hinter sich haben, sind grundsätzlich immer dienstuntauglich. Gemäss den Ausführungen des Oberfeldarztes [2] sind Patienten, die mit Methadon oder anderen Substanzen substituiert sind - auch bei guter sozialer Integration und fehlenden somatischen Begleiterscheinungen - dienstuntauglich, da die pharmakologischen Wirkungen der Substanzen als Gefahrenpotential bestehen bleiben.

Komplementärmedizin: Übernimmt die Militärversicherung die Behandlungskosten für Komplementärmedizin?

Seit diesem Jahr übernimmt die Militärversicherung als Pflichtleistung ebenfalls die Kosten aus folgenden komplementärmedizinischen Bereichen:

- Klassische Akupunktur;

- Anthroposophische Medizin;

- Chinesische Medizin;

- Homöopathie;

- Neuraltherapie;

- Phytotherapie.

Die Militärversicherung hält fest, dass diese Liste von komplementärmedizinischen Therapieformen abschliessend ist, und dass

- die aufgeführten Therapieformen von eidgenössisch diplomierten Ärzten durchgeführt werden müssen;

- die Verrechnung nach Zeitaufwand gemäss Arzttarif erfolgen muss;

- die Heilmittelkosten nur dann übernommen werden, wenn die Heilmittel bei der IKS registriert sind. 


\section{Anlaufstellen}

Auskunftspersonen in der Sektion MAD für administrative oder medizinische Fragen sind:

Sektionschef: Dr. med. R. Huber, Tel. 03132427 28, Fax 03132427 63, E-mail: mad@gst.admin.ch

Zuständige Kreisärzte (nach Regionen aufgeteilt):

Romandie, Oberwallis, FR, Seeland, Laufental:

Kreisarzt I: Dr. med. G. Daucourt, Tel. 0313242732

Innerschweiz, ZG, LU, AG, SO, BS, BL, BE:

Kreisarzt II: M. Büchler, med. pract., Tel. 0313242730

ZH, Ostschweiz, GR, TI:

Kreisarzt III: Dr. med. F. Frey, Tel. 0313242731

\section{Médecin civil - Service militaire}

\section{Quelles sont les questions les plus fréquemment posées à la section du service médico-militaire du Grasan par les médecins civils exerçant en cabinet privé?}

Etat-major général, groupe des affaires générales, section du service médico-militaire

\section{Introduction}

La section du service médico-militaire (SMM) tient lieu de centrale d'informations dans le domaine médico-militaire, d'une part pour les médecins d'école et de troupe, mais aussi pour les militaires et leurs médecins traitants civils. (Remarque: le terme «médecins civils" s'applique aux personnes des deux sexes).

La section SMM du Groupe des affaires sanitaires comprend trois bureaux d'arrondissements, avec chacun un médecin d'arrondissement, le bureau du recrutement et le chef de la section.

Vous trouverez ci-après des réponses succinctes aux questions qui nous sont le plus souvent posées.

Correspondance:

Dr Rolf Huber

EMG, Grasan

Section SMM

CH-3003 Berne

\section{Literatur}

1 Frey F. Diensttauglichkeit. Schweiz Ärztezeitung 1999; 80(19):1180-3.

2 Eichenberger P. Drogen - Diensttauglichkeit. Umgang mit Drogenkonsumenten im Militärdienst. Kriterien zur Beurteilung der Diensttauglichkeit. Schweiz Ärztezeitung 2000; 81(10):506-8.

Les thèmes abordés sont répartis entre les questions administratives et les problèmes touchant la médecine militaire.

\section{Questions administratives}

Déplacement d'un service: une recrue ou un militaire doit déplacer son service pour raisons médicales. A qui faut-il envoyer le certificat médical? Il faut faire une distinction entre soldats, sous-officiers et officiers.

Pour les soldats, une demande de déplacement de service pour raisons médicales doit être envoyée par l'intermédiaire du service chargé des convocations (pour les troupes cantonales, le commandement d'arrondissement concerné; pour les troupes fédérales, l'Etat-major général, Groupe du personnel de l'armée).

Pour les sous-officiers et officiers, la demande de déplacement du service doit être envoyée au commandant d'unité concerné.

Dans les deux cas, les certificats médicaux doivent être joints sous pli fermé à la demande de déplacement du service. Dans tous les cas, ils doivent être présentés par les services susnommés à la section SMM pour appréciation. Le cas échéant, celle-ci présente, de son côté, la proposition de dispense au service chargé de convoquer. Lorsque le certificat médical n'a pas été mis sous pli fermé, le service chargé de convoquer peut, dans les cas jugés clairs, (par ex. fracture du tibia, hospitalisations etc.) prononcer une dispense du service sans en référer à la section SMM.

Lors de demandes adressées à court terme (15 jours avant le service) il est préférable d'envoyer en sus une copie de la demande de déplacement du service et du certificat médical directement à la section SMM, de manière à abréger la procédure administrative. 
Licenciement médical du service militaire (inaptitude au service): à qui faut-il envoyer les certificats médicaux lorsqu'un militaire doit être licencié de l'armée? Comment faut-il rédiger un tel certificat? Les certificats qui ne concernent pas l'aptitude à faire service (pour un service imminent) mais l'aptitude au service en général doivent être envoyés directement à la section SMM.

La section SMM convoque ensuite le militaire en question devant une commission de visite sanitaire (CVS) ou, lorsque les cas sont clairs et bien documentés, elle prononce in absentia la décision "inapte» par la CVS du Groupe des affaires sanitaires.

Les certificats médicaux sont toujours appréciés par les médecins d'arrondissement de la section SMM et doivent être rédigés en bonne et due forme. Tous les collaborateurs de la section ayant accès aux dossiers médicaux sont soumis au secret professionnel.

La section SMM et les commissions de visite sanitaire se réjouissent de recevoir de la part du médecin traitant un certificat détaillé. Contrairement à ce que l'on pourrait penser, la plupart des commissions de visite sanitaire ne procèdent pas à un examen médical complet des militaires qui leur sont envoyés, mais apprécient les patients uniquement sur la base des documents dont elles disposent et en fonction de l'entretien personnel.

Pour cette raison, le certificat médical devrait comprendre au moins une brève anamnèse, le diagnostic exact, l'évolution de l'affection, le traitement et le pronostic. Dans la mesure où ils sont disponibles, les résultats d'examens médicaux spéciaux (p.ex. les rapports radiologiques, les résultats de laboratoire et, pour les certificats psychiatriques, les résultats du test psychologique) y seront joints. Une proposition du médecin traitant au sujet de l'aptitude au service sera également la bienvenue. Des renseignements sur les capacités sportives sont aussi une contribution à l'appréciation de l'aptitude au service. Il faut relever ici que les commissions de visite sanitaire doivent tenir compte du certificat médical, mais elles prennent librement leur décision en ce qui concerne l'aptitude au service.

Du fait que les dossiers sont archivés selon le numéro AVS, son indication sur le certificat médical nous facilite grandement la tâche.

Critères d'appréciation: quels sont les critères d'appréciation de l'aptitude au service militaire et de l'inaptitude au service?

Les critères définissant l'aptitude au service se trouvent dans le document militaire confidentiel «Nosologia Militaris». Cette documentation ne peut donc pas, pour cause de classification, être remise aux médecins civils.

La "Bulletin des médecins suisses" [1] a toutefois publié des extraits de cette documentation ainsi que les directives générales. Cet article peut être demandé comme tirage à part auprès de la section SMM.
Prise en charge des frais: un certificat médical ou une expertise doivent être établis pour un militaire. Qui doit en assumer les frais?

En principe, lorsqu'un militaire souhaite que soit établi un certificat médical, c'est à lui de prendre en charge le coût de ce certificat.

Lorsque le Grasan ordonne un examen complémentaire, les confrères ayant un cabinet privé reçoivent une demande écrite (avec une formule de compensation). Dans ce cas, les frais sont à la charge du Grasan. Le médecin civil peut établir une facture selon le tarif de l'AA/AM/AI.

Médecin civil engagé comme médecin de troupe: un médecin civil est chargé de fournir l'assistance médicale à la troupe. Quelles possibilités a-t-il de s'informer sur la tâche qui l'attend?

Il arrive qu'en raison du manque d'un médecin de troupe, l'on fasse appel à des médecins civils pour l'assistance médicale de la troupe en temps de paix.

La troupe annonce à la section SMM les médecins civils qui assument cette tâche, afin que nous puissions envoyer à ces collègues un classeur avec une documentation détaillée. Si cette procédure ne fonctionne pas automatiquement, les médecins civils peuvent demander les documents en question directement à la section SMM.

Le classeur de documentation susmentionné contient des documents sur la fonction du médecin civil dans le service du médecin de troupe (par ex. les directives du médecin en chef de l'armée du 29.12.1998 concernant la prise en charge médicale de troupes par des médecins civils), dans lesquels sont précisées aussi bien les tâches du médecin civil que la question de l'indemnisation. Le classeur de documentation contient aussi les critères d'appréciation de l'aptitude au service (la "Nosologia Militaris») ainsi qu'une sélection des formules les plus importantes.

Frais de traitement: traitement de militaires par des médecins civils pendant le service. Qui prend en charge les frais?

Les militaires présentant des problèmes médicaux durant les prestations de service sont tenus de s'annoncer au médecin de troupe. Les prestations de service militaire englobent aussi le congé général et le congé privé. C'est seulement en cas d'urgence durant le congé que le militaire s'adressera à un médecin civil.

L'assurance militaire couvre les frais médicaux ambulatoires et d'hospitalisation qui surviennent durant le service. Les séjours hospitaliers sont calculés pour tous militaires selon le tarif de la division commune. Des séjours en division semi-privée ou privée sont subsidiairement pris en charge par les caisses maladies privées, pour autant qu'une assurance complémentaire existe. Dans ce cas, une assurance privée suspendue pendant le service militaire doit être réintroduite. 
Tableau 1

Inaptitude à faire service après implantation de matériel d'ostéosynthèse.

\begin{tabular}{ll}
\hline Localisation & Délai de carence (mois) \\
\hline Bras & $6-12$ \\
\hline Avant-bras & $4-6$ \\
\hline Colonne vertébrale & 12 \\
\hline Cuisse & $12-18$ \\
\hline Jambe et pied & $6-12$ \\
\hline
\end{tabular}

\section{Tableau 2}

Inaptitude à faire service après ablation du matériel d'ostéosynthèse.

\begin{tabular}{ll}
\hline Localisation & Délai de carence (mois) \\
\hline Extrémité supérieure & 3 \\
\hline Cuisse & 6 \\
\hline Jambe et pied & 3 \\
\hline
\end{tabular}

Il arrive de temps à autre qu'en fin de semaine, des militaires s'adressent au médecin civil aussi pour des problèmes bénins ou pour avoir un second avis. Les renseignements pris auprès de l'assurance militaire ont révélé que celle-ci prend également en charge ces consultations.

Pour ce genre de consultation, les certificats médicaux doivent être remis au patient, à l'attention du médecin de troupe.

Retrait de l'arme: l'arme personnelle d'un patient souffrant de troubles psychiques et pouvant être dangereux pour lui-même et pour autrui doit lui être retirée. Comment le médecin traitant va-t-il procéder?

Dans ce cas, le médecin traitant doit demander à la police locale de procéder à la confiscation de l'arme.

La police déposera l'arme en question à l'arsenal concerné contre remise d'une pièce justificative.

Simultanément, il faut adresser à la section SMM une proposition de dispense provisoire du service militaire ou de déclaration d'inaptitude au service.

La section SMM décide ensuite, sur la base du dossier médical, si l'arme doit être retirée définitivement au militaire ou si on pourra la lui restituer après un certain temps.

Service civil: comment faut-il rédiger un certificat médical dans lequel un militaire est déclaré apte à accomplir uniquement le service civil?

Le militaire qui désire accomplir un service civil doit être impérativement apte au service dans l'armée. C'est seulement à cette condition qu'il peut déposer une demande pour accomplir un service civil.

Contrairement à d'autres armées (p.ex. l'Allemagne), la Suisse ne connaît pas le libre choix entre le service militaire et le service civil. En Suisse, seul celui qui, pour d'authentiques raisons de conscience, ne peut pas faire de service militaire sera autorisé à accomplir du service civil. Les raisons de conscience seront appréciées par une commission d'admission non-médicale!

En résumé, les critères médicaux sont valables pour l'appréciation de l'aptitude au service. L'aptitude au service est la condition pour formuler une demande de service civil. Pour l'admission dans le service civil, des critères éthiques restent valables, mais non pas des faits d'ordre médical.

Remarque: les notions de "service civil» et de "service de la protection civile" prêtent fréquemment à confusion. Le service civil décrit ci-dessus est un service de remplacement du service militaire. Le service de la protection civile est obligatoire après l'accomplissement du service militaire obligatoire, respectivement après la libération du service militaire pour des raisons médicales.

\section{Questions médicales}

Orthopédie: y a-t-il des directives concernant l'aptitude à faire service à la suite d'interventions orthopédiques?

La doctrine de base est qu'à la suite d'interventions chirurgicales, l'aptitude à faire service repose sur les recommandations du chirurgien ayant effectué l'opération ou du médecin prenant en charge le traitement ultérieur.

La présence de matériel d'ostéosynthèse ne constitue pas en soi une raison suffisante contre-indiquant l'aptitude à faire service.

S'il n'existe pas de recommandations concrètes du médecin traitant, le médecin de troupe se référera aux directives concernant le délai de carence après une implantation (tab. 1), respectivement après une ablation du matériel d'ostéosynthèse (tab. 2).

Après l'échéance de ces délais de carence, le militaire est à nouveau apte à faire service sans restriction. Pour que cette condition soit remplie, il ne doit résulter aucun trouble fonctionnel ni atteinte des tissus mous (par exemple, cicatrices handicapantes).

Si un doute subsiste, il est recommandé de prendre contact avec la section SMM pour mettre au point la procédure à suivre.

Epileptiques: les épileptiques qui suivent un traitement médicamenteux efficace sont-ils aptes au service dans certaines fonctions?

En principe, les épileptiques ne sont pas aptes au service, à l'exception des patients dont les crises d'épilepsie se sont limitées à l'enfance.

Même si un épileptique bien accommodé peut, dans la vie civile, obtenir un permis de conduire, l'aptitude au service n'est pas remplie.

Test HIV positif: les patients séropositifs sont-ils en principe aptes au service?

Les conscrits et les recrues séropositifs qui sont sous traitement sont obligatoirement inaptes au service. 
Chez les militaires dont l'instruction de base est achevée, la situation doit être appréciée de façon plus différenciée. L'appréciation dépend de l'évolution médicale, du traitement, de la fonction militaire et de l'attitude du militaire. Nous vous renvoyons de nouveau à l'article paru dans le bulletin des médecins suisses [1].

Patients traités à la méthadone: les militaires sous méthadone et avec un pronostic favorable sur le plan médical et social, peuvent-ils être aptes au service dans des cas exceptionnels?

Les patients qui consomment des drogues dures, qui sont en cure de sevrage (y compris le programme de substitution) ou qui ont terminé une cure sont toujours inaptes au service. Selon le médecin en chef de l'armée [2], les patients traités à la méthadone ou avec d'autres substances sont inaptes au service, et cela même s'ils sont bien intégrés socialement et s'ils ne présentent pas d'effets secondaires somatiques, car les effets pharmacologiques des substances constituent un danger potentiel.

Médecines complémentaires: l'assurance militaire prend-elle en charge les coûts des traitements effectués pour les médecines complémentaires?

Depuis cette année, l'assurance militaire est tenue de prendre en charge les frais provenant des médecines complémentaires suivantes:

- acuponcture classique,

- médecine anthroposophique,

- médecine chinoise,

- homéopathie,

- thérapie neurale,

- phytothérapie.
L'assurance militaire précise que cette liste est considérée comme définitive et que:

- les traitements en question doivent être appliqués par des médecins détenteurs du diplôme fédéral;

- la facturation en fonction du temps consacré doit être établie selon le tarif médical;

- les coûts des médicaments ne sont pris en charge que si ceux-ci sont enregistrés auprès de l'OICM.

\section{Informations}

A la section SMM, les personnes suivantes peuvent fournir des renseignements d'ordre médical ou administratif:

Chef de section. Dr R. Huber, tél. 03132427 28, fax

03132427 63, e-mail: mad@gst.admin.ch

Médecins d'arrondissement (selon les régions):

Romandie, Haut-Valais, FR, Seeland, Laufental: Méd ar I: Dr G. Daucourt, tél. 0313242732

Suisse centrale, ZG, LU, AG, SO, BS, BL, BE: Méd ar II: M. Büchler, méd. prat., tél. 0313242730

$\mathrm{ZH}$, Suisse orientale, GR, TI:

Méd ar III: Dr F. Frey, tél. 0313242731

\section{Références}

1 Frey F. Diensttauglichkeit. Schweiz Ärztezeitung 1999; 80(19):1180-3.

2 Eichenberger P. Drogen - Diensttauglichkeit. Umgang mit Drogenkonsumenten im Militärdienst. Kriterien zur Beurteilung der Diensttauglichkeit. Schweiz Ärztezeitung 2000; 81(10):506-8. 